\title{
Trapping the Transition State in a $[2,3]$-Sigmatropic Rearrangement by Applying Pressure
}

\author{
Sourabh Kumar ${ }^{1}$ and Tim Stauch*,1,2,3 \\ ${ }^{1}$ University of Bremen, Institute for Physical and Theoretical Chemistry, Leobener Straße NW2, D-28359 Bremen, Germany \\ 2 Bremen Center for Computational Materials Science, University of Bremen, Am Fallturm 1, D-28359 Bremen, Germany \\ ${ }^{3}$ MAPEX Center for Materials and Processes, University of Bremen, Bibliothekstraße 1, D-28359 Bremen, Germany
}

\begin{abstract}
Transition states are of central importance in chemistry. While they are, by definition, transient species, it has been shown before that it is possible to "trap" transition states by applying stretching forces. We here demonstrate that the task of transforming a transition state into a minimum on the potential energy surface can be achieved by using hydrostatic pressure. We apply the computational eXtended Hydrostatic Compression Force Field (XHCFF) approach to the educt of a [2,3]-sigmatropic rearrangement in both static and dynamic calculations and find that the five-membered cyclic transition state of this reaction becomes a minimum at pressures in the range between 100 and $150 \mathrm{GPa}$. Slow decompression leads to a 70:30 mix of the product and the educt of the sigmatropic rearrangement. Our findings are discussed in terms of geometric parameters and electronic rearrangements throughout the reaction. We speculate that the trapping of transition states by using pressure is generally possible if the transition state of a chemical reaction has a more condensed geometry than both the educt and the product, which paves the way for new ways of initiating chemical reactions.
\end{abstract}

* Corresponding author. Email: tstauch@uni-bremen.de 
The rearrangement of allylic sulfoxides to allylic sulfenate esters is a popular example of $[2,3]-$ sigmatropic rearrangements. ${ }^{[1-3]}$ This type of reaction is also known as Mislow-Evans rearrangement ${ }^{[4-11]}$ and is widely used in asymmetric synthesis due to the possibility of chirality transfer from the carbon bearing the sulfoxide group to the alcohol resulting from a subsequent reaction with an appropriate thiophile (Figure 1A). Hence, the Mislow-Evans rearrangement is a popular tool in the synthesis of bioactive substances and natural products like terpenes, ${ }^{[12]}$ vernolepin, ${ }^{[13,14]}$ amphidinol $3,{ }^{[15]}$ and pyrenolide D. ${ }^{[16]}$

A<smiles>CC=CC(C)S(C)=O</smiles><smiles>CC1=BC(C)(C)C(C)=C1</smiles>

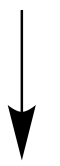<smiles>CC=CC(C)O</smiles><smiles>[124IH]</smiles><smiles>CCC(C)C</smiles><smiles>CC=CC(C)OSC</smiles>

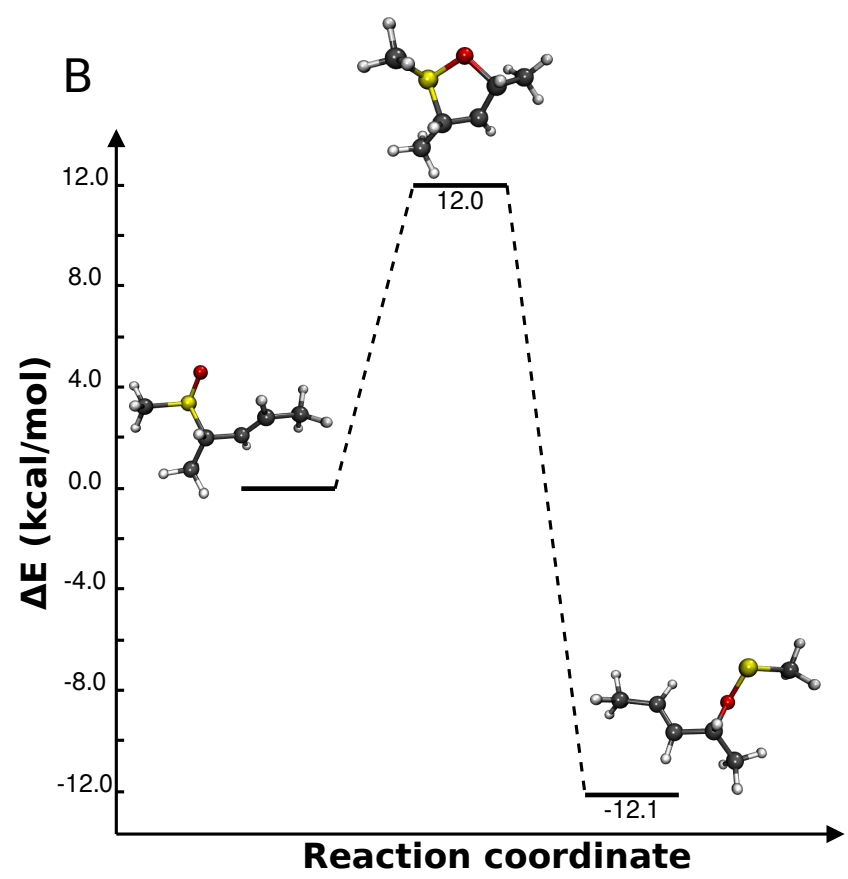

C

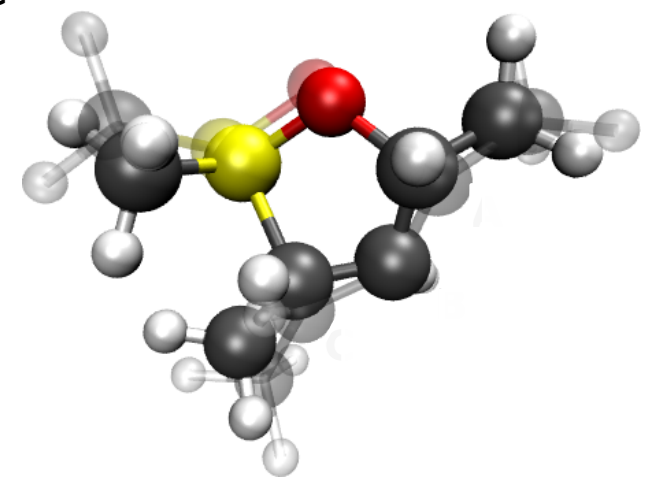

Figure 1: (A) Schematic representation, including labels of carbon atoms, and (B) energy profile of the Mislow-Evans rearrangement, calculated at the B3LYP/6-31G(d) level of theory. (C) Superimposed geometries of the structure found at $120 \mathrm{GPa}$ (solid), calculated with X-HCFF, and the cyclic transition state of the Mislow-Evans rearrangement without pressure (transparent). 
The transition state of the Mislow-Evans rearrangement has attracted considerable attention, because it determines the stereochemistry of the reaction. ${ }^{[17]}$ It has been found that the Mislow-Evans rearrangement proceeds via a five-membered cyclic transition state (Figure 1A). ${ }^{[6,11,17-19]}$ By using quantum chemical methods, we here demonstrate that the cyclic transition state of the Mislow-Evans rearrangement becomes a minimum under pressures between 100 and $150 \mathrm{GPa}$, which is a pressure range that is accessible with modern diamond-anvil cell technology. ${ }^{[20]}$ While the "trapping" of a transition state has been achieved before with mechanical force, ${ }^{[21]}$ to the best of our knowledge this is the first time that hydrostatic pressure has been used for this purpose. The possibility of transforming a transition state into a minimum on the potential energy surface paves the way for isolating and characterizing this transient chemical species and will more broadly open up new possibilities in chemical synthesis.

Several quantum chemical methods for the application of hydrostatic pressure to molecules during geometry optimizations have been reported. ${ }^{[22-29]}$ We apply the eXtended Hydrostatic Compression Force Field (X-HCFF) approach ${ }^{[28]}$ in this paper, since this method has been used successfully to predict structural parameters and chemical reactions in the GPa regime. Furthermore, Born-Oppenheimer Molecular Dynamics (BOMD) simulations can be conducted

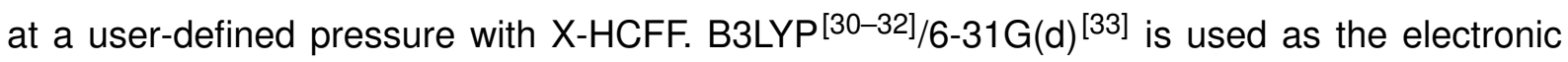
structure method throughout. Further computational details can be found in the Supporting Information.

While, according to our calculations, the cyclic transition state of the model Mislow-Evans reaction considered in this paper (Figure 1B) lies $12.0 \mathrm{kcal} / \mathrm{mol}$ above the educt, geometry optimization with X-HCFF at a pressure of $120 \mathrm{GPa}$ yields a five-membered cyclic structure (Figure 1C). This compressed geometry is remarkably close to the pressure-free transition state, with a mean RMSD of only $0.3 \AA$ between the ring atoms in the compressed and the pressure-free geometries (Table S1). While transition states have been found to disappear under pressure, ${ }^{[27]}$ leading to the formation of the product of a chemical reaction with no further energy input, our calculations demonstrate that pressure can be used to transform a transition state into a minimum on the potential energy surface. Despite the intriguing notion that a transition state can be isolated, this finding is intuitive, since the transition state of the MislowEvans rearrangement has a more condensed geometry than both the educt and the product. $\mathrm{A}$ preference of the transition state geometry is therefore expected at elevated pressures.

To investigate the formation of the five-membered ring in the model Mislow-Evans reaction under pressure more closely, we conducted ten individual BOMD simulations, in which we increased the pressure in increments of $10 \mathrm{GPa}$ every 96.8 fs. Two representative distances, 

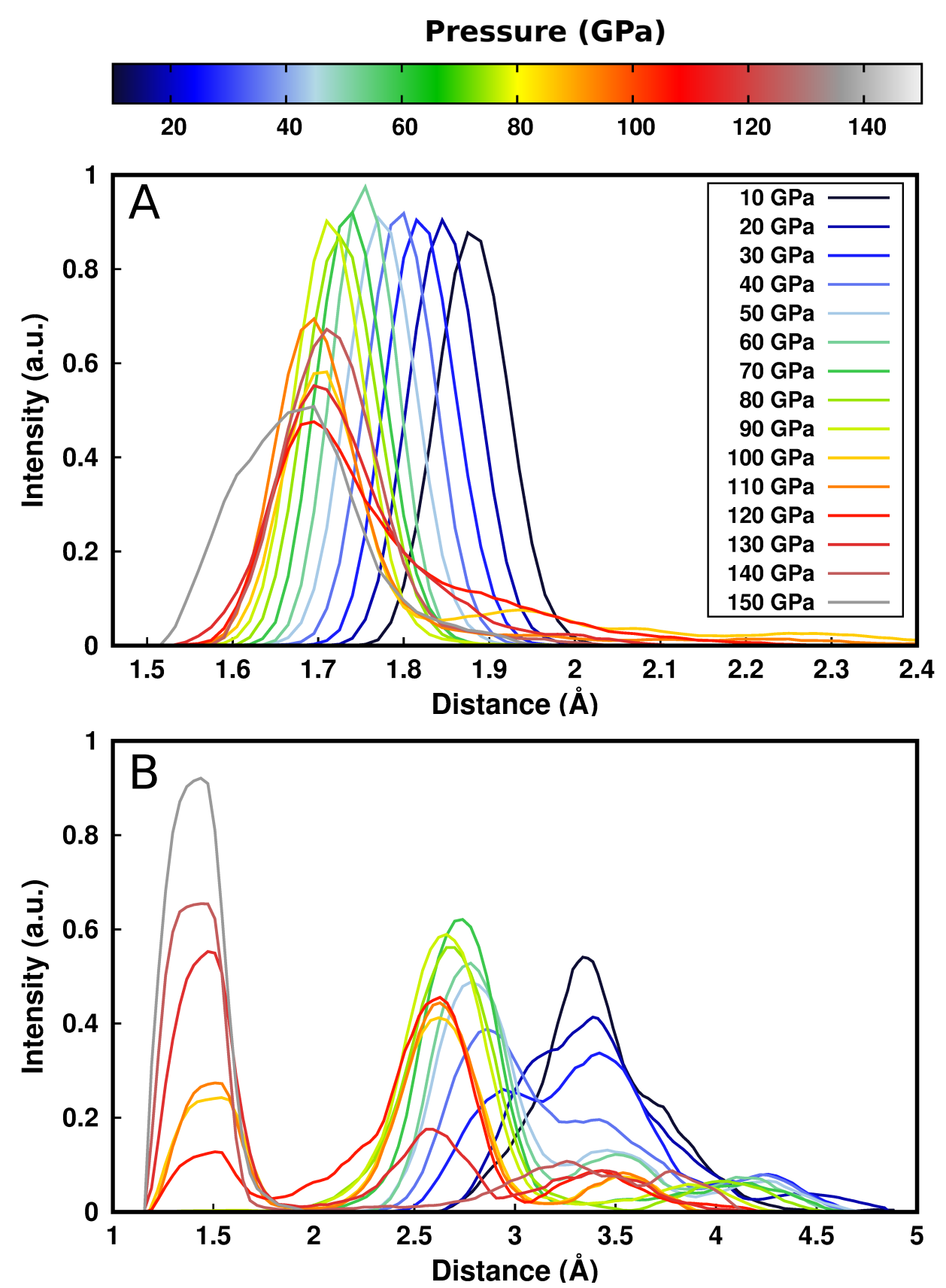

Figure 2: Normalized distances $\mathrm{S}-\mathrm{C}_{A}(\mathrm{~A})$ and $\mathrm{C}_{C}-\mathrm{O}(\mathrm{B})$ for each individual $10 \mathrm{GPa}$ simulation window averaged over all trajectories in the pressure range between 0 and $150 \mathrm{GPa}$.

$\mathrm{S}-\mathrm{C}_{A}$ and $\mathrm{C}_{C}-\mathrm{O}$ (cf. Figure $1 \mathrm{~A}$ for the labels), were averaged over all ten simulations for each $10 \mathrm{GPa}$ simulation window up to the maximum applied pressure. In agreement with chemical intuition, the $S-C_{A}$ bond shortens with increasing pressure (Figure $2 A$ ). Up to approx. $90 \mathrm{GPa}$, the $S-C_{A}$ bond length decreases fairly linearly with increasing pressure, and, since pressure is proportional to force, this observation shows that the compression of the $\mathrm{S}-\mathrm{C}_{A}$ bond is a harmonic process throughout a remarkably large pressure range.

Turning to the $\mathrm{C}_{C}-\mathrm{O}$ distance (Figure $2 \mathrm{~B}$ ), the distribution initially only broadens up to a pressure of $20 \mathrm{GPa}$, since $\mathrm{C}_{C}$ and $\mathrm{O}$ are not bonded at first. An intermediate, slightly com- 
pressed geometry with a $\mathrm{C}_{C}-\mathrm{O}$ distance between 2.5 and $3 \AA$ is subsequently generated, followed by the gradual generation of cyclic species in which $C_{C}$ and $O$ are bonded, as evidenced by the accumulation of structures with a $\mathrm{C}_{C}-\mathrm{O}$ distance of approx. $1.5 \AA$ at pressures above $100 \mathrm{GPa}$.

The distance $\mathrm{C}_{C}-\mathrm{O}$, which forms during the Mislow-Evans rearrangement, and $\mathrm{S}-\mathrm{C}_{A}$, which breaks, are shown in Figure 3 as a function of time for two representative BOMD trajectories. In the first trajectory (Figure $3 \mathrm{~A}$ ), formation of the five-membered ring can be observed at approx. $1.05 \mathrm{ps}(120 \mathrm{GPa})$, marked by a sharp decrease in the $\mathrm{C}_{C}-\mathrm{O}$ distance. Subsequently, slow decompression leads to the rupture of the $\mathrm{S}-\mathrm{C}_{A}$ bond, yielding the reaction product. While in the second trajectory (Figure $3 \mathrm{~B}$ ) the formation of the five-membered ring can be observed at a pressure of $120 \mathrm{GPa}$ as well, decompression in this case leads to the recovery of the educt due to the rupture of the $\mathrm{C}_{C}-\mathrm{O}$ bond and not the $\mathrm{S}-\mathrm{C}_{A}$ bond. Similar observations are made for other structural parameters involved in the formation of the five-membered ring (Figures S1 and S2).

The possibility to either form the product or to recover the educt is the expected behavior for a system that is held artificially at the transition state, since it can move in either direction when the constraint is lifted, depending on the instantaneous nuclear velocities that are present upon decompression. In seven out of the ten simulations, the formation of the product is observed, whereas the educt is recovered in the remaining three simulations. This observation lends further evidence that a transition state was isolated using pressure. The pressure required to form the five-membered ring ranges between 100 and $150 \mathrm{GPa}$, depending on the trajectory. Details on each individual trajectory are given in the Supporting Information (Table S2).

The formation of the five-membered ring and its transformation to either the product or the educt of the Mislow-Evans rearrangement upon decompression can also be understood in terms of changes in Mayer bond orders ${ }^{[34,35]}$ throughout the BOMD simulations (Figure 4). While the $\mathrm{C}_{B}=\mathrm{C}_{C}$ bond loses its double bond character upon formation of the five-membered ring, the bond order of the $\mathrm{C}_{A}-\mathrm{C}_{B}$ bond increases, signifying an increase in double-bond character. In the trajectory in which the product of the Mislow-Evans reaction is formed upon decompression (Figure 4A), these changes are permanent. In addition, the bond order analysis reveals the rupture of the $S-C_{A}$ bond and the formation of the $C_{C}-O$ bond, completing the rearrangement. Contrarily, the decrease in $\mathrm{S}-\mathrm{C}_{A}$ bond order and the formation of the $\mathrm{C}_{C}-\mathrm{O}$ bond are only transitory in the trajectory that recovers the educt upon decompression (Figure 4B). As soon as the five-membered ring dissociates upon decompression and the educt forms, the bond orders return to their initial values in this trajectory. 

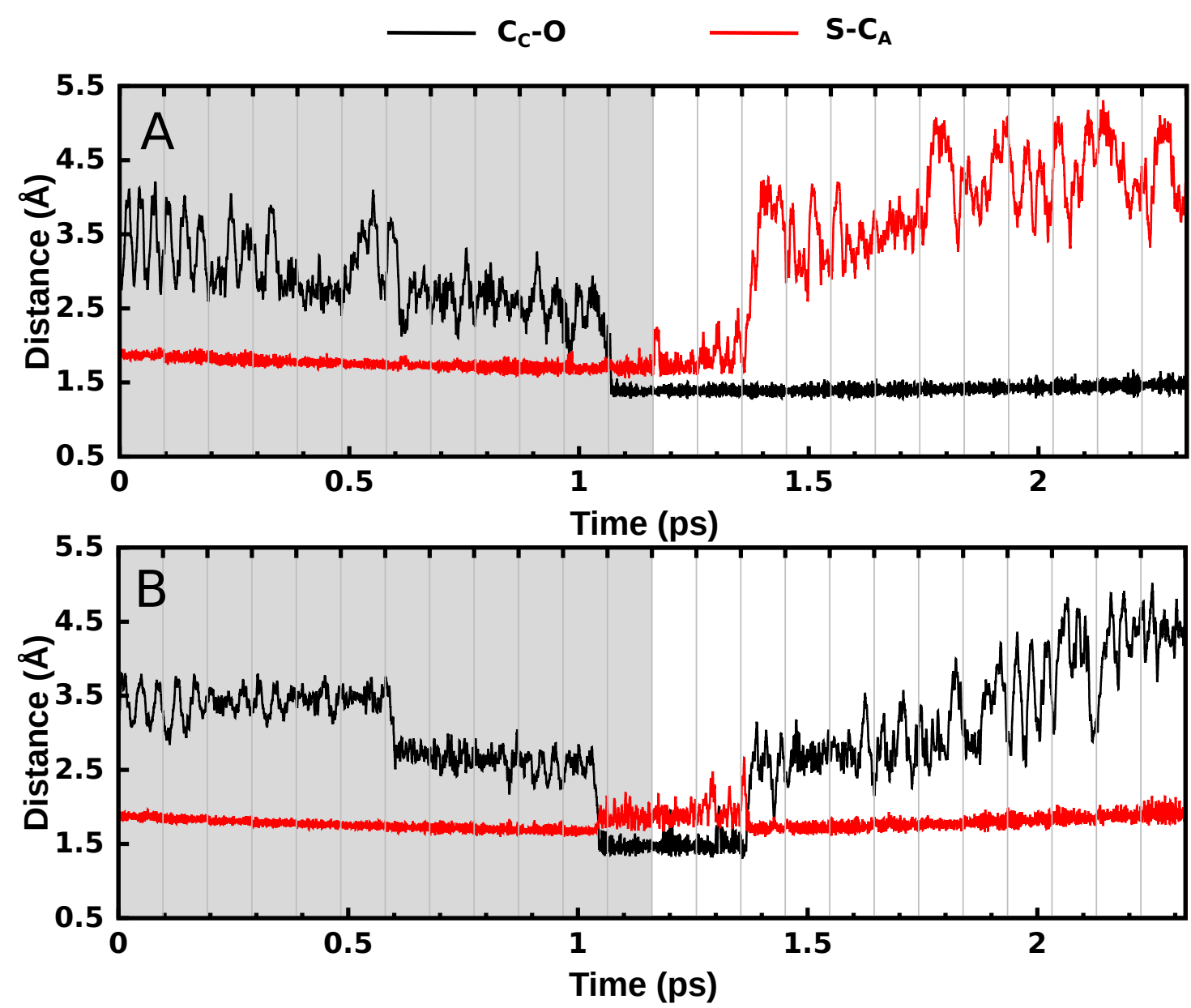

Figure 3: Time evolution of the distances $\mathrm{C}_{C}-\mathrm{O}$ (black line) and $\mathrm{S}-\mathrm{C}_{A}$ (red line) for two representative $B O M D$ simulations of the model system under hydrostatic pressure in which the formation of the product (A) or of the educt $(B)$ of the Mislow-Evans rearrangement is observed, respectively. Gray shaded areas signify increasing compression, whereas white areas denote decompression. The pressure was changed by $10 \mathrm{GPa}$ at each of the gray vertical lines.

Analogous effects can be observed in the propagation of electron densities at the bond critical points throughout the BOMD trajectories (Figure S3): In addition to an overall increase in electron density with increasing pressure, which is due to the compressed nuclear scaffold and the shortened bonds, shifts in electron density mark the formation of the five-membered cyclic structure at high pressure and its transformation to either the product or the educt of the Mislow-Evans rearrangement upon decompression.

The quantum chemical calculations and BOMD simulations presented in this study demonstrate that the five-membered cyclic transition state of a model Mislow-Evans rearrangement can be "trapped" by applying hydrostatic pressures in the range between 100 and $150 \mathrm{GPa}$. Decompression leads to the formation of the product of the rearrangement in seven out of ten trajectories, whereas the educt is recovered in the remaining three simulations. Our future studies will focus on the influence of environmental factors like chemical substitution ${ }^{[36]}$ or the solvent in a multiscale simulation setup. We hope that our calculations spark the interest in 

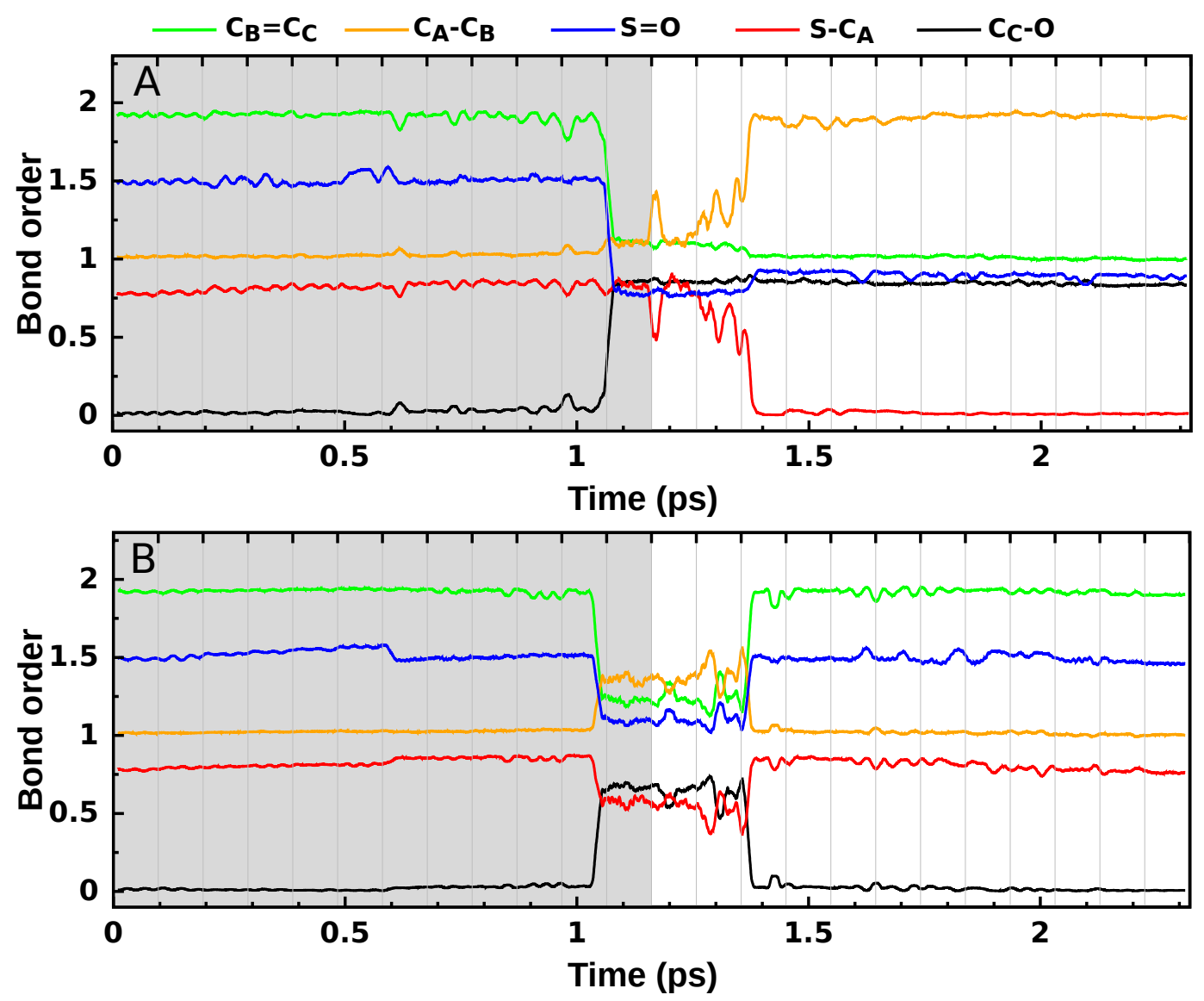

Figure 4: Time series plot of the Mayer bond orders of the bonds in the five-membered ring throughout two representative BOMD trajectories in which the product $(A)$ or the educt $(B)$ of the Mislow-Evans rearrangement is formed, respectively.

experimental studies on the barochemical isolation of transitory species in sigmatropic rearrangements and other chemical reactions. We speculate that transition states can generally be isolated by using pressure if the transition state geometries are more condensed than both the educt and the product of the reaction. 


\section{References}

[1] M. Reggelin, "[2, 3]-sigmatropic rearrangements of allylic sulfur compounds," Sulfur-Mediated Rearrangements II, pp. 1-65, 2007.

[2] F. Tang, Y. Yao, Y. Xu, and C. Lu, "Diastereoselective Aza-Mislow-Evans Rearrangement of N -Acyl tert -Butanesulfinamides into $\alpha$-Sulfenyloxy Carboxamides," Angew. Chem., vol. 130, no. 47, pp. 15809-15812, 2018.

[3] I. Colomer, M. Velado, R. Ferna, D. Pradilla, and A. Viso, "From Allylic Sulfoxides to Allylic Sulfenates : Fifty Years of a NeverEnding [ 2 , 3 ] -Sigmatropic Rearrangement," Chem. Rev., vol. 117, pp. 14201-14243, 2017.

[4] D. R. Rayner, E. G. Miller, P. Bickart, A. J. Gordon, and K. Mislow, "Mechanisms of Thermal Racemization of Sulfoxides," J. Am. Chem. Soc., vol. 88, no. 13, pp. 3138-3139, 1966.

[5] E. G. Miller, D. R. Rayner, and K. Mislow, “Thermal Rearrangement of Sulfenates to Sulfoxides1,” J. Am. Chem. Soc., vol. 88, no. 13 , pp. 3139-3140, 1966.

[6] P. Bickart, F. W. Carson, J. Jacobus, E. G. Miller, and K. Mislow, "The Thermal Racemization of Allylic Sulfoxides and the Interconversion of Allylic Sulfoxides and Sulfenates. Mechanism and Stereochemistry1,2," J. Am. Chem. Soc., vol. 90, no. 18, pp. 4869-4876, 1968.

[7] D. A. Evans, G. C. Andrews, and C. L. Sims, "Reversible 1,3 Transposition of Sulfoxide and Alcohol Functions. Potential Synthetic Utility," J. Am Chem. Soc., vol. 93, no. 19, pp. 4956-4957, 1971.

[8] D. A. Evans and G. C. Andrews, "Nucleophilic Cleavage of Allylic Sulfenate Esters. Mechanistic Observations," J. Am Chem. Soc., vol. 94, no. 10, pp. 3672-3674, 1972.

[9] D. A. Evans, G. Andrews, T. T. Fujfmoto, and D. Wells, "Stereoselective Synthesis of Trisubstituted Olefins," Tetrahedron Lett., vol. 16, pp. 1389-1392, 1973.

[10] D. A. Evans, G. C. Andrews, T. T. Fujimoto, and D. Wells, "The application of allylic sulfoxide anions as vinyl anion equivalents. A general synthesis of allylic alcohols.," Tetrahedron Lett., vol. 16, pp. 1385-1388, 1973.

[11] R. Tang and K. Mislow, "Rates and Equilibria in the Interconversion of Allylic Sulfoxides and Sulfenates1," J. Am Chem. Soc., vol. 92, no. 7, pp. 2100-2104, 1970.

[12] Y. Masaki, K. Hashirnoto, K. Sakuma, and K. Kaji, "Facile Regio- and Stereo-specific Allylic Oxidation of gem-Dimethyl Olefins via Addition of Benzenesulphenyl Chloride. Synthesis of Allylic Oxygenated Terpenes," J. CHEM. SOC. PERKIN TRANS, vol. 1, pp. 1289-1295, 1984.

[13] H. lio, M. Isobe, T. Kawai, and T. Goto, "Total Synthesis of Vernolepin. 2.1 Stereocontrolled Synthesis of ( \pm )-Vernolepin," J. Am Chem. Soc., vol. 101, no. 20, pp. 6076-6081, 1979.

[14] F. Zutterman, H. D. Wilde, R. Mungheer, P. D. Clercq, and M. Vandewalle, "A total synthesis of (+-)-Vernolepin," Tetrahedron, vol. 35, pp. 2389-2396, 1979.

[15] J. de Vicente, J. R. Huckins, and S. D. Rychnovsky, "Synthesis of the C31-C67 Fragment of Amphidinol 3," Angew. Chem., vol. 118, no. 43, pp. 7416-7420, 2006.

[16] K. M. Engstrom, M. R. Mendoza, M. Navarro-villalobos, and D. Y. Gin, "Total Synthesis of (+ ) -Pyrenolide D **," Angew. Chem. Int. Ed., vol. 40, no. 6, pp. 1128-1130, 2001.

[17] Reinhard W. Hoffmann, "Stereochemistry of [2,3]Sigmatropic Rearrangements," Angew. Chem. Int. Ed. Engl., vol. 18, no. 8, pp. 563-640, 1979.

[18] D. K. Jones-hertzog and W. L. Jorgensen, "Elucidation of Transition Structures and Solvent Effects for the Mislow - Evans Rearrangement of Allylic Sulfoxides," J. Am Chem. Soc., no. 11, pp. 9077-9078, 1995.

[19] F. Freeman, R. M. Bathala, J. E. Cavillo, A. C. Huang, T. K. Jackson, A. Z. Lopez-Mercado, S. Phung, J. Suh, and J. Diego O. Valencia, "[2,3]-Sigmatropic Rearrangements of Hydrogen and Alkyl 3-Propenyl Sulfoxides: A Computational Study," Int. J. Quantum Chem., vol. 106, pp. 2390-2397, 2006. 
[20] M. Li, Q. Zhang, Y. N. Zhou, and S. Zhu, "Let spiropyran help polymers feel force!," Progress in Polymer Science, vol. 79, pp. 26-39, 2018.

[21] J. M. Lenhardt, M. T. Ong, R. Choe, C. R. Evenhuis, T. J. Martinez, and S. L. Craig, "Trapping a diradical transition state by mechanochemical polymer extension," Science, vol. 329, no. 5995, pp. 1057-1060, 2010.

[22] T. Stauch, "Quantum chemical modeling of molecules under pressure," Int. J. Quantum Chem., vol. 121, no. 3, pp. 1-8, 2020.

[23] G. Subramanian, N. Mathew, and J. Leiding, "A generalized force-modified potential energy surface for mechanochemical simulations," Journal of Chemical Physics, vol. 143, no. 13, 2015.

[24] T. Stauch, R. Chakraborty, and M. Head-Gordon, "Quantum Chemical Modeling of Pressure-Induced Spin Crossover in Octahedral Metal-Ligand Complexes," ChemPhysChem, vol. 20, pp. 2742- 2747, 2019.

[25] R. Cammi, V. Verdolino, B. Mennucci, and J. Tomasi, "Towards the elaboration of a QM method to describe molecular solutes under the effect of a very high pressure," Chem. Phy., vol. 344, no. 1-2, pp. 135-141, 2008.

[26] R. Cammi, "A new extension of the polarizable continuum model: Toward a quantum chemical description of chemical reactions at extreme high pressure," J. Comput. Chem., vol. 36, no. 30, pp. 2246-2259, 2015.

[27] Z. Chen, J. A. Mercer, X. Zhu, J. A. Romaniuk, R. Pfattner, L. Cegelski, T. J. Martinez, N. Z. Burns, and Y. Xia, "Mechanochemical unzipping of insulating polyladderene to semiconducting polyacetylene.," Science, vol. 357, no. 6350, pp. 475-479, 2017.

[28] T. Stauch, "A mechanochemical model for the simulation of molecules and molecular crystals under hydrostatic pressure," $J$. Chem. Phys., vol. 153, no. 13, p. 134503, 2020.

[29] M. Scheurer, A. Dreuw, E. Epifanovsky, M. Head-Gordon, and T. Stauch, "Modeling Molecules under Pressure with Gaussian Potentials," J. Chem. Theory Comput., vol. 17, pp. 583-597, 2021.

[30] A. D. Becke, "Correlation energy of an inhomogeneous electron gas: A coordinate-space model," J. Chem. Phys., vol. 88, no. 2, pp. 1053-1062, 1988.

[31] C. Lee, W. Yang, and R. G. Parr, "Development of the Colic-Salvetti correlation-energy formula into a functional of the electron density," Phys. Rev. B, vol. 37, no. 2, pp. 785-789, 1988.

[32] A. D. Becke, "A new mixing of Hartree-Fock and local density-functional theories," J. Chem. Phys., vol. 98, no. 2, pp. 1372$1377,1993$.

[33] W. J. Hehre, R. Ditchfield, and J. A. Pople, "Self - Consistent Molecular Orbital Methods . XII . Further Extensions of Gaussian - Type Basis Sets for Use in Molecular Orbital Studies of Organic Molecules Published by the AIP Publishing Articles you may be interested in Selfconsistent molecular orbit," J. Chem. Phys., vol. 56, pp. 2257-2261, 1972.

[34] I. Mayer, "Charge, bond order and valence in the ab initio SCF theory," Chem. Phys. Lett., vol. 97, no. 3, pp. 270-274, 1983.

[35] I. Mayer, "On Bond Orders and Valences in the Ab Znitio Quantum Chemical Theory," Int. J. Quantum Chem., vol. 29, no. 1, pp. 73-84, 1986.

[36] S. Kumar and T. Stauch, "The activation efficiency of mechanophores can be modulated by adjacent polymer composition," RSC Adv., vol. 11, no. 13, pp. 7391-7396, 2021. 